\title{
Quantum Quenches, Thermalization and Many-Body Localization
}

\author{
Elena Canovi, ${ }^{1}$ Davide Rossini, ${ }^{2}$ Rosario Fazio, ${ }^{2}$ Giuseppe E. Santoro, ${ }^{1,3,4}$ and Alessandro Silva ${ }^{3}$ \\ ${ }^{1}$ International School for Advanced Studies (SISSA), Via Bonomea 265, I-34136 Trieste, Italy \\ ${ }^{2}$ NEST, Scuola Normale Superiore, and Istituto Nanoscienze - CNR, Pisa, Italy \\ ${ }^{3}$ International Centre for Theoretical Physics (ICTP), P.O. Box 586, I-34014 Trieste, Italy \\ ${ }^{4}$ CNR-INFM Democritos National Simulation Center, Via Bonomea 265, I-34136 Trieste, Italy
}

\begin{abstract}
We conjecture that thermalization following a quantum quench in a strongly correlated quantum system is closely connected to many-body delocalization in the space of quasi-particles. This scenario is tested in the anisotropic Heisenberg spin chain with different types of integrability-breaking terms. We first quantify the deviations from integrability by analyzing the level spacing statistics and the inverse participation ratio of the system's eigenstates. We then focus on thermalization, by studying the dynamics after a sudden quench of the anisotropy parameter. Our numerical simulations clearly support the conjecture, as long as the integrability breaking term acts homogeneously on the quasiparticle space, in such a way as to induce ergodicity over all the relevant Hilbert space.

PACS numbers: 75.10.Jm, 72.15.Rn, 05.45.Mt
\end{abstract}

\section{INTRODUCTION}

The understanding of ergodicity and thermalization in quantum systems is one of the most intriguing problems in quantum physics. Starting with the 1929 paper of John von Neumann $\underline{1}$, various attempts have been made towards the characterization of ergodic behavior in quantum systems ${ }^{2}-\underline{5}$ and the establishment of a link with the notion of quantum chaos 4.5 . Theoretical interest in these issues resurfaced periodically $\underline{\underline{6}}$ until very recently, when an experimental study of the non-equilibrium dynamics of a quasi-one-dimensional condensate clearly demonstrated the lack of thermalization/ergodicity in a quantum many-body system ${ }^{7}$. The attribution of this observation to quantum integrability generated a lot of interest on its connections with ergodicity and thermalization in strongly-correlated quantum systems ${ }^{8,9}$.

The simplest setting to study the relaxation of manybody systems is to consider an abrupt change in time of one of the control parameters, i.e., a quantum quench. At long times after the quench, the lack of thermalization in an integrable system can be seen as a consequence of the sensitivity to the specifics of the initial state encoded in the values of the constants of motion $\underline{*}$. This lead to the proposal of describing the time-averaged steady state reached after a quench by keeping track of the initial value of all the constants of motion through a general-

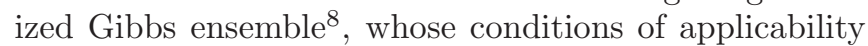
and drawbacks have been extensively tested $\underline{\underline{9}}$. In turn, if the system is far enough from the integrable limit, thermalization is generally expected to occur, as numerically confirmed in many circumstances $\underline{10}-12$.

This qualitative picture, although very appealing, leaves a number of important questions unanswered. It is not yet clear what is the nature of the integrable/nonintegrable transition. Moreover, as it was shown in Ref. 13, it appears that even an integrable system could look "thermal", depending on the observable which is analyzed. Operators which are non-local in the quasiparticles of the system may behave thermally, while local operators do not. How to reconcile all these observations under a unifying framework?

The purpose of this paper is to show that the underlying mechanism governing the thermalization of manybody systems (and its relation to integrability) is that of many-body localization in Fock space $\underline{14}-16$. We organize the paper as follows. In Sec. III after explaining in details the key concept of many-body localization, we discuss qualitatively our conjecture on thermalization following a quantum quench, i.e., on the role played by many-body localization. The model under investigation is then introduced in Sec. III In the following sections we discuss our results: first of all we address the spectral properties of the model and use them to characterize the localized and delocalized regimes (Sec. IV]) we then focus on the quench dynamics, providing evidence of the connection between delocalization and thermalization according to standard statistical mechanics predictions (Sec. (V) . Finally, in Sec. V1] we draw our conclusions.

\section{MANY-BODY LOCALIZATION AND QUANTUM QUENCHES}

In this section we discuss a qualitative scenario connecting the physics of thermalization after a quantum quench to the phenomenon of many-body localization. In order to do so, let us first lie down a few basic facts about many-body localization, as originally discussed in the context of transport of interacting electrons in random potentials 14,16 . In absence of electron-electron interactions, the physics of disordered electron systems can be understood in terms of the standard Anderson localization phenomenology: extended wave functions correspond to finite zero-temperature conductivity while localized states correspond to vanishing conductivity. The Anderson localization-delocalization transition is therefore naturally associated to a metal-insulator quantum phase transition. Notice that since localized and extended states cannot mix in the spectrum, the latter is a 
sequence of bands of extended and localized states separated by mobility edges.

The nature of the spectrum and of the eigenstates can change drastically if electron-electron interactions are taken into account. In particular, it has been recently shown 14 that even when all single-particle states are localized, the presence of electron-electron interactions and inelastic collisions can result in an insulatorto-metal transition as the temperature of the system is raised above a certain critical value $T_{c}$. Such a phase transition can be thought of as a many-body localizationdelocalization transition, occurring at the level of manybody eigenstates 14,16 . Indeed, the presence of the manybody localization transition at finite temperature implies the existence of a many-body mobility edge at an energy scaling extensively with system size and separating localized many-body states, at low energies, from extended many-body states, at higher energies 14 .

Many-body localization is a rather general concept which does not necessarily refer to real space. A standard example in this sense comes from the physics of quasi-particle relaxation in quantum dots. Here the concepts of localization and delocalization find their natural applicability in Fock space, where all many-body eigenstates are defined $\underline{16}$. Similar ideas were also employed to analyze the mixing of vibrational modes due to anharmonicity in molecules $\frac{17}{17}$. Along these lines, it was recently realized $\frac{18-20}{2}$ that the many-body localizationto-delocalization transition discussed above should be deeply connected to the main subject of this work, the physics of integrability-breaking. More specifically, let us think of an integrable model (having well defined quasi-particles) as a multidimensional lattice in which each point, identified by the occupations $n(k)$ of the various quasi-particle modes, represents an eigenstate $\left|\Psi_{\alpha}\right\rangle=\left|\left\{n_{\alpha}(k)\right\}\right\rangle$ (see Fig. (1). The space of these states (the quasi-particle space) is an obvious generalization of the standard Fock space. As long as states are localized in quasi-particle space ${ }^{21}$, one expects the system to behave as integrable: any initial condition spreads into few sites, maintaining strong memory of the initial state. On the other hand, once a strong enough integrability-breaking perturbation hybridizing the various states $\left|n_{\alpha}(k)\right\rangle$ is applied, a consequent delocalization in quasi-particle space will occur (see Fig. 1). A tendency towards ergodicity is expected in this case.

In this paper we aim at establishing a close connection between the physics of the localization-delocalization transition/crossover, occurring in quasi-particle space in the presence of an integrability-breaking term, and the physics of thermalization. In order to do so, we focus on a specific class of non-equilibrium protocols on which thermalization can be studied, the so-called quantum quenches. In the present context they are defined through the time-dependent Hamiltonian

$$
\mathcal{H}(t) \equiv \mathcal{H}_{0}[g(t)]+\mathcal{H}_{i b},
$$

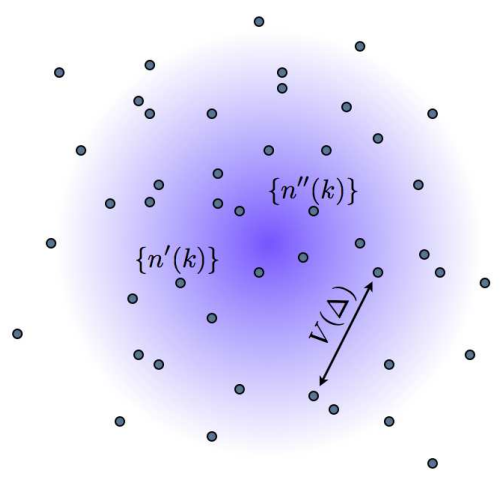

Figure 1: (color online). A cartoon of the quasi-particle space. For an integrable model all states, represented by the occupations of quasi-particles $\{n(k)\}$, are localized. An integrability-breaking perturbation introduces hopping matrix elements $V$ among different sites, which hybridize, provided $\mid E\left(\left\{n^{\prime}(k)\right\}-E\left(\left\{n^{\prime \prime}(k)\right\} \mid \leq V\right.\right.$. For strong perturbations this may lead to delocalization of wave functions among all points in quasiparticle space in a microcanonical energy shell.

where:

$$
g(t)=\left\{\begin{array}{l}
g_{0} \text { for } t<0 \\
g \text { for } t \geq 0
\end{array}\right.
$$

The time-dependent part of the Hamiltonian $\mathcal{H}_{0}[g(t)]$ is integrable, while $\mathcal{H}_{i b}$ is the integrability-breaking term. We then ask ourselves the following question: what are the characteristics that many-body eigenstates should have in order for the system to thermalize and behave ergodically?

The answer to an analogous question for semiclassical quantum chaotic systems was conjectured by M. Berry in $1977^{22}$ and later employed by M. Srednicki to discuss thermalization in a (non-integrable) gas of interacting particles ${ }^{5}$. Inspired by these seminal papers, we propose that for generic many-body systems thermalization will occur whenever the eigenstates of the system become diffusive in microcanonical shells defined in quasi-particle space. These diffusive states correspond to the intuitive expectation that in an ergodic state any initial state is allowed to diffuse into all states in a micro-canonical energy shell, generating a cascade of all possible lower energy excitations 23 . The purpose of the remaining sections is to test this proposal on the dynamics of a concrete integrable model.

\section{THE MODELS}

In order to corroborate the scenario proposed above, we now study in detail the dynamics after a quench of an anisotropic Heisenberg chain subject to various forms of integrability-breaking perturbations. The anisotropic 
Heisenberg (XXZ) spin-1/2 chain is defined by:

$$
\mathcal{H}_{0}\left(J_{z}\right)=\sum_{i=1}^{L-1}\left[J\left(\sigma_{i}^{x} \sigma_{i+1}^{x}+\sigma_{i}^{y} \sigma_{i+1}^{y}\right)+J_{z} \sigma_{i}^{z} \sigma_{i+1}^{z}\right],
$$

where $\sigma_{i}^{\alpha}(\alpha=x, y, z)$ denote the spin-1/2 Pauli matrices on site $i, J$ the coupling strength, while $J_{z}$ the $z$-anisotropy intensity. Units of $\hbar=k_{B}=1$ are used throughout the paper; $J=1$ is taken as the energy scale. This Hamiltonian is integrable by Bethe Ansatz, and exhibits two gapped phases, ferromagnetic $\left(J_{z}<-1\right)$ and antiferromagnetic $\left(J_{z}>1\right)$, separated by a critical region $-1 \leq J_{z} \leq 1$, with $J_{z}$-dependent critical exponents 24 and quasi-long-range-order in the $x y$ spin-plane.

As for the integrability-breaking perturbation, we consider different cases, with or without disorder terms in the Hamiltonian, which can be expressed in the form:

$$
\mathcal{H}_{i b}=\sum_{i} \Delta_{i} \mathcal{O}_{i}
$$

where $\Delta_{i}$ is the amplitude (possibly site-dependent) of an additional few-body term $\mathcal{O}_{i}$. This few-body term may act on a single site $i$ (e.g., onsite magnetic field), or on a few sites centered around $i$ (e.g., nearest or nextto-nearest neighbor couplings). In particular, we break integrability by either adding: $(I)$ a random magnetic field in the $z$-direction; $(I I)$ random $J_{z}$ couplings; $(I I I)$ random or $(I V)$ uniform next-nearest neighbor $z z$ couplings, according to:

$$
\mathcal{H}_{i b}=\left\{\begin{array}{l}
\Delta \sum_{i=1}^{L} h_{i} \sigma_{i}^{z} \\
\Delta \sum_{i=1}^{L-1} h_{i} \sigma_{i}^{z} \sigma_{i+1}^{z} \\
\Delta \sum_{i=1}^{L-2} h_{i} \sigma_{i}^{z} \sigma_{i+2}^{z} \\
\Delta \sum_{i=1}^{L-2} \sigma_{i}^{z} \sigma_{i+2}^{z}
\end{array}\right.
$$

For cases $(I)-(I I)-(I I I)$ integrability-breaking is induced by the disorder, $h_{i} \in[-1,1]$ being random numbers, while in case $(I V)$ disorder is not invoked. In general, disordered systems allow for a better statistical analysis, due to the possibility of averaging over randomness. One might argue that such averages are strictly required in order to reproduce our findings about thermalization. This is not the case, since we have found analogous qualitative conclusions in all the four cases discussed above: integrability-breaking is the only crucial requirement for our mechanism of thermalization to set in.

We first address the spectral properties of the model, and subsequently consider a sudden quench of the anisotropy parameter $J_{z} \equiv g$. The total magnetization $S^{z}=\sum_{i} \sigma_{i}^{z}$ is a conserved quantity, hence we restrict to the sector $S^{z}=0$. Nonetheless, due to the involvement in such non-equilibrium dynamics of a considerable part of the spectrum, standard (both analytic and numerical) renormalization group techniques are eventually doomed to failure. We therefore resort to exact numerical diagonalization of systems with up to 16 spins.
While the zero-temperature phase-diagram in presence of disorder is well established 25], the high-temperature phase-diagram has been conjectured to be composed of two phases, a non-ergodic many-body localized phase (in real space) at $\Delta>\Delta^{\text {crit }}$, and an ergodic one at $\Delta<\Delta^{\text {crit }}$; in the case $(I), \Delta^{\text {crit }} \sim 6 \div 8$ at $J_{z}=1$ (in our units) $\frac{18,19}{10}$. The results presented below indicate the presence of a second non-ergodic localized phase (in quasi-particle space) for $\Delta$ close to zero that crosses over to the ergodic phase upon increasing $\Delta$. The fate of this crossover in the thermodynamic limit and the eventual value of the critical $\Delta^{*}$ are yet to be determined ${ }^{26}$.

We start by characterizing deviations from integrability in terms of the many-body level statistics and of the properties of the eigenstates. A well defined transition from Poisson (Integrable) to Wigner-Dyson statistics (non-Integrable) is closely associated to the localized/diffusive character of eigenstates in quasi-particle space. Using this characterization, we then show that the non-thermal-to-thermal transition in the dynamics is directly connected to the localization/delocalization transition in quasi-particle space. In particular, by looking at the asymptotics of spin-spin correlation functions, we discuss how thermalization is linked to the emergence of diffusive eigenstates in quasi-particle space. This also allows us to discuss, in a broad context, the relationship between locality of observables in quasi-particle space and the corresponding behavior.

\section{SPECTRAL PROPERTIES}

Let us first concentrate on the spectral properties of the Hamiltonian for a given value of the anisotropy $g=$ $J_{z}$. In the following we show data for $J_{z}=0.5$. We have explicitly checked that, changing $J_{z}$ to a different value within the critical region of the XXZ model $\left(\left|J_{z}\right| \leq 1\right)$, does not qualitatively affect the scenario discussed and our conclusions.

\section{A. Level Spacing Statistics}

The statistics of the energy levels represents a key feature of the spectrum of a generic quantum system, since it is a good indicator of the presence of integrability. Both in semiclassical and in many-body systems, integrable systems have levels that tend to cluster, eventually crossing when a parameter in the Hamiltonian is varied. On the other hand, in non-integrable systems the levels are correlated in such a way as to avoid crossings. A quantitative way to characterize these tendencies is through the Level Spacing Statistics (LSS) 27 , i.e., the probability distribution $P(s)$ that the energy difference between two adjacent levels $s_{n} \equiv E_{n+1}-E_{n}$ (normalized to the average level spacing) falls in the interval $[s, s+d s]$. In a typical integrable system one finds a Poissonian (P) LSS:

$$
P_{\mathrm{P}}(s)=e^{-s} .
$$


On the other hand, for non-integrable systems one expects random matrix theory to apply, leading to a Wigner-Dyson (WD) distribution, where level repulsion shows up in $\lim _{s \rightarrow 0} P(s) \sim s^{\gamma}$. More specifically, for systems as the one considered here, which preserve one anti-unitary symmetry (invariance under time-reversal), the statistics is given by a Gaussian Orthogonal Ensemble 27 (at low energy spacings one has the characteristic behavior $\gamma=1)$ :

$$
P_{\mathrm{WD}}(s)=\frac{\pi s}{2} e^{-\frac{\pi s^{2}}{4}} .
$$

In our case the system undergoes a transition from Poissonian [Eq. (50)] to Wigner-Dyson [Eq. (6)] LSS upon increasing the non-integrable perturbation $\Delta$, which for finite-size systems takes the form of a smooth crossover. This can be faithfully quantified by means of the Level Spacing Indicator (LSI) $\eta$ :

$$
\eta \equiv \frac{\int_{0}^{s_{0}}\left[P(s)-P_{P}(s)\right] d s}{\int_{0}^{s_{0}}\left[P_{W D}(s)-P_{P}(s)\right] d s}
$$

where $P(s)$ is the probability distribution function of the level spacing between neighboring levels, while $s_{0} \approx$ 0.4729 is the first intersection point of $P_{\mathrm{P}}(s)$ and $P_{\mathrm{WD}}(s)$. The LSI is zero for systems with a Poisson distribution $P_{\mathrm{P}}$ of the spacings and one if the distribution is WignerDyson $P_{\mathrm{WD}}$. Below we will study the LSI in two different ways: $(i)$ as a function of the energy eigenvalues in individual microcanonical shells $\left[\eta_{\mathrm{w}}(E)\right]$, using Eq. (7) with $P_{[E, E+W]}(s)$ as the level statistics computed in the energy window $[E, E+W] ;(i i)$ cumulatively $\left[\eta_{\mathrm{c}}(E)\right]$, as a function of the energy eigenvalues below a given threshold, with $P_{\left[E_{0}, E\right]}(s)$ as the level statistics of eigenvalues with excitation energy less than $E$, with respect to the ground state energy $E_{0}$.

\section{Results for the LSI}

The LSS in the XXZ model with an integrabilitybreaking perturbation has been the subject of various studies in the literature $\underline{28,29}$. Here we are not interested in a complete characterization of it, but rather on elucidating under which conditions, and in which regions of the energy spectrum, the model behaves according to the WD statistics, i.e., $\eta \approx 1$ according to our definition. We point out that the WD distribution of Eq. (6) is obtained for non-integrable systems with only a timereversal symmetry. In all our simulations we considered open boundary conditions, fixed the sector of zero magnetization, and added a very small magnetic field on the first site of the chain, in such a way as to work in a subspace without any other unwanted symmetry.

All the four types of integrability-breaking perturbation behave quite in the same way, the only difference being for case $(I V)$, where fluctuations are more consistent, due to the absence of disorder averaging (to reduce
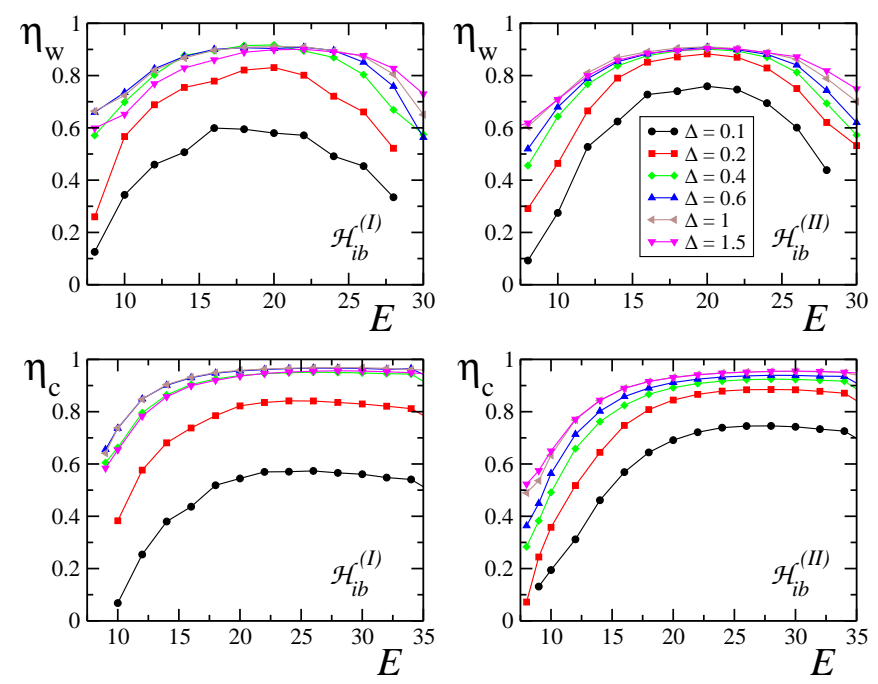

Figure 2: (color online). Level spacing indicator for the XXZ model (3) with non-integrable perturbations (I) (left panels) and $(I I)$ (right panels). The upper frames show $\eta_{\mathrm{w}}(E)$, while the lower ones display $\eta_{\mathrm{c}}(E)$. Data are for $L=14$ sites with different values of the integrability-breaking perturbation $\Delta$. The LSI $\eta_{\mathrm{w}}(E)$ is evaluated in a microcanonical shell of width $W=2$. Averages are performed over $10^{3}$ disorder instances. In order to exactly recover Poisson and GOE statistics in the two integrable and non-integrable limits, we performed an unfolding of the energy spectrum for each instance, according to standard techniques adopted in quantum chaos 27 .

fluctuations, one should consider energy spectra of larger systems; however, the exact diagonalization technique intrinsically imposes severe size limitations). As an explicative example, in Fig. 2 we plot both $\eta_{\mathrm{w}}(E)$ and $\eta_{\mathrm{c}}(E)$ for the XXZ model with a random $z$-field, $(I)$ (left panels), and with random $J_{z}$ couplings, $(I I)$ (right panels). We observe that, fixing the system size, if $\Delta$ is progressively increased, the value of $\eta$ also increases, until it reaches, in the middle of the energy band, a value close to 1 (for $\Delta \sim 1$, in our units and at $L=14$ ). For $\Delta \gtrsim 1, \eta$ decreases again towards small values, since for $\Delta \gg J_{z}$ the system turns into a trivial classically integrable model 29 . Only in the middle of the spectrum the system appears to exhibit level repulsion, while this is not the case in the low- or high-energy spectrum ${ }^{28}$. This is more evident from the cumulative LSI $\eta_{c}$; here one can notice that, for sufficiently strong perturbations and at low energies, $\eta_{c}$ is an increasing function of $E$, until it saturates around its maximal value.

\section{B. Inverse participation ratio}

After a spectral characterization of integrability through the LSS, we come back to the characterization of the eigenstates. In the region where a Poissonian LSS is observed, the eigenstates are expected to be localized in 
quasiparticles space, while if WD is seen, the eigenstates are expected to be delocalized. The proper tool to quantitatively characterize the properties of the eigenstates and their delocalization is the so called Inverse Participation Ratio (IPR) $\stackrel{27,30}{ }$. The IPR on a normalized pure state $|\psi\rangle$ is a basis-dependent quantity, defined by:

$$
\xi(|\psi\rangle)=\frac{1}{N}\left(\sum_{n=1}^{N}|\langle n \mid \psi\rangle|^{4}\right)^{-1},
$$

where $\{|n\rangle\}$ is the reference basis of the Hilbert space. If a state is a uniform superposition of $n_{\text {st }}$ basis states, the corresponding contribution to $\xi$ is of order $n_{\text {st }}$.

We will focus on the IPR of the system eigenstates, evaluated of two types of basis: $(i)$ the site $(S)$ basis $\left|n_{S}\right\rangle=\left|\sigma_{1} \cdots \sigma_{L}\right\rangle\left(\sigma_{i}= \pm 1\right)$, composed by the eigenstates of $\sigma_{i}^{z}$, which is often referred to as the "computational basis"; (ii) the integrable (I) basis, composed by the eigenstates of the integrable model (3) in absence of the perturbation terms: $\Delta=0$. Analogously to the LSI, we can compute the IPR over microcanonical shells around a given energy value $E$. Notice that, if an eigenstate is localized in quasi-particle space, we expect the inverse participation ratio computed in the integrable basis to be $\xi_{I} \simeq O(1)$. Conversely, if an eigenstate is a diffusive superposition with random phases and similar amplitudes of $N$ eigenstates of the integrable model, then $\xi_{I} \simeq N$. Below we will use these facts to characterize localization and delocalization in quasi-particle space.

\section{Results for the IPR}

In Fig. 3 we show the inverse participation ratio for the XXZ model with the integrability-breaking terms $(I)$ and $(I I)$ (as for the LSI, results are not qualitatively different if different perturbations are considered). Looking at the IPR in the site basis (lower panels), as long as $\Delta$ is increased we observe a general tendency to a localization (the IPR peak value decreases). This is coherent with the fact that the states of the computational basis are exactly the eigenstates of the system for $\Delta \gg J_{z}$. On the other hand, as depicted in the upper panels, the IPR in the integrable basis behaves rather differently. In particular, it provides a clear signature of the fact that eigenstates delocalize with increasing values of the disorder $\Delta$.

In order to better understand the nature of the delocalization induced by the integrability-breaking term, let us compare $\xi$ with the number of states $N_{[E, E+W]}$ in the relevant microcanonical shell $[E, E+W]$, where $W \sim V$ is the typical matrix element of the integrabilitybreaking perturbation ( $V \approx 2 \Delta$ in this case). For small $\Delta, \xi_{I} \ll N_{[E, E+W]}$ (see Fig. 4, upper panels), indicating that the eigenstates are still close to those of the integrable system and the degree of delocalization of the system is very low. On the contrary, when $\Delta \simeq 1$ (Fig. 4] lower panels) we observe that $\xi_{I} \simeq N_{[E, E+W]}$. In this case, the perturbation is able to hybridize nearly all the
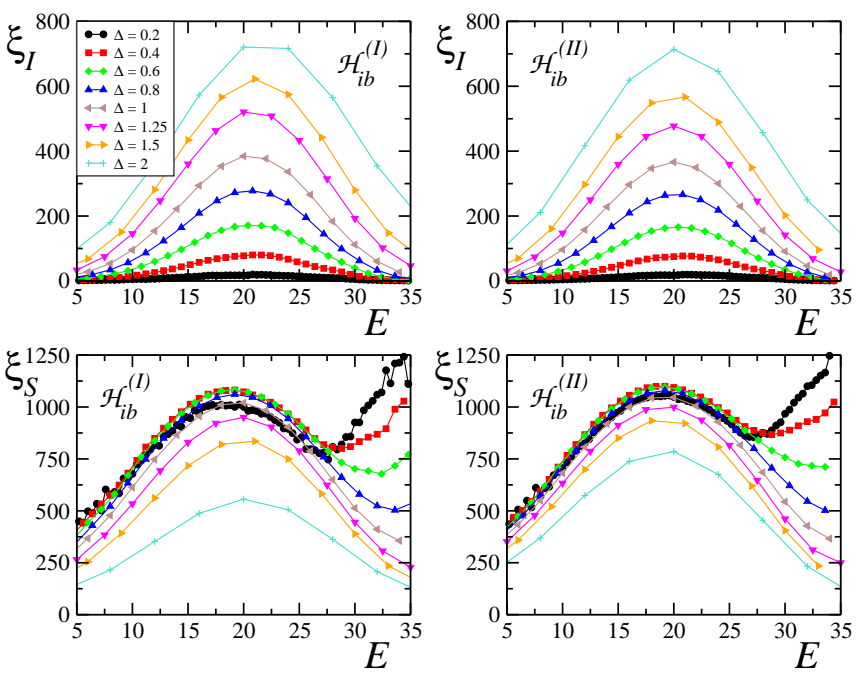

Figure 3: (color online). Inverse participation ratio for the XXZ model (3) with non-integrable perturbations (I) (left panels) and (II) (right panels). Data are for $L=14$ sites and different values of the perturbation strength $\Delta$. The IPR is evaluated in a microcanonical shell of width $W=2 \Delta$, in the integrable (upper frames) and in the site (lower frames) basis. Data are averaged over all the system eigenstates in the appropriate energy window, and over $10^{2}$ disorder realizations.

quasiparticles states within the microcanonical energy shell. As we will see in the next section, this is the key ingredient for the system to thermalize. Notice that in this context the low-lying eigenstates are rather peculiar: this part of the spectrum, which contains very few states as compared to the center, has closely Poissonian statistics and is characterized by large fluctuations of statistical quantities.

\section{DYNAMICS AFTER THE QUENCH}

It is now time to use the information we obtained about spectral statistics and eigenstates to study the relation between many-body localization and thermalization. We will look at the dynamics following a sudden quench of the anisotropy parameter $g \equiv J_{z}$ from $J_{z 0}$ at $t \leq 0$ to $J_{z} \neq J_{z 0}$ at $t>0$, as described in Eq. (2). As we did for the spectral properties, we will show data for systems where the anisotropy is quenched toward $J_{z}=0.5$. Different values of such $J_{z}$ do not qualitatively affect the scenario. The system is initially prepared in the ground state $\left|\psi_{0}\right\rangle$ of $\mathcal{H}\left(J_{z 0}\right)$, so that its (conserved) energy with respect to the final Hamiltonian $\mathcal{H}\left(J_{z}\right)$ is $E_{0}=\left\langle\psi_{0}\left|\mathcal{H}\left(J_{z}\right)\right| \psi_{0}\right\rangle$. For growing values of $J_{z 0}$, the state $\left|\psi_{0}\right\rangle$ tends towards the classical antiferromagnetic Néel state, and $E_{0} / L$ saturates to a constant value, slightly below the middle of the spectral band, thus implying that a quench generally involves only a fraction of the eigenstates of the final Hamiltonian. 

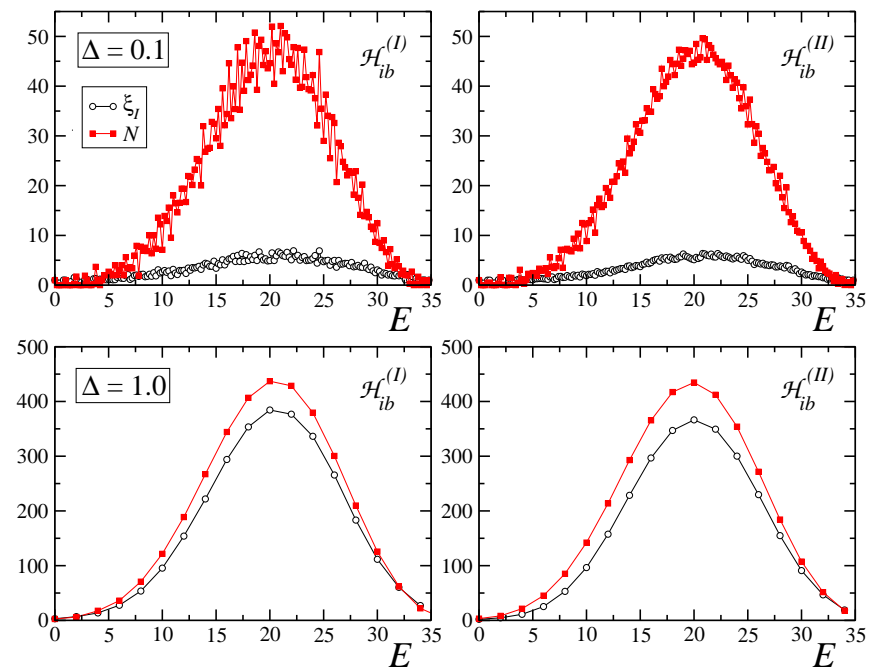

Figure 4: (color online). IPR in the integrable basis $\xi_{I}$ at $\Delta=0.1$ (upper panels), and at $\Delta=1$ (lower panels), compared to the number of states $N$ in an energy window of width $W=2 \Delta$. Results are shown for the integrability-breaking terms $(I)$ (left panels) and $(I I)$ (right panels). Notice however that all other cases display an identical qualitative behavior. In particular, the disorder is not required to observe the hybridization of the quasiparticle states within the microcanonical energy shell for the case of large $\Delta$.

\section{A. Effective temperature}

Contrary to local quenches, the work done on the system by changing the anisotropy from $J_{z 0}$ to $J_{z}$ is extensive. It is then interesting to ask, after a quench involving an extensive injection of energy $E_{0}-E_{\mathrm{gs}} \propto L\left[E_{\mathrm{gs}}\right.$ being the ground state energy of $\left.\mathcal{H}\left(J_{z}\right)\right]$, if the subsequent longtime evolution of the system is effectively described by an equilibrium dynamics governed by $\mathcal{H}\left(J_{z}\right)$. In view of a plausible equivalence between a microcanonical (fixed $\left.E_{0}\right)$ and a canonical equilibrium description of such a long-time dynamics, it is meaningful to define, as in previous instances ${ }^{13}$, an effective temperature $T_{\text {eff }}$ for the system out of equilibrium. We compute $T_{\text {eff }}$ by equating the micro-canonical energy $E_{0}=\left\langle\psi_{0}\left|\mathcal{H}\left(J_{z}\right)\right| \psi_{0}\right\rangle$ to the canonical ensemble average

$$
E_{0} \equiv\left\langle\mathcal{H}\left(J_{z}\right)\right\rangle_{T_{\text {eff }}}=\operatorname{Tr}\left[\rho\left(T_{\text {eff }}\right) \mathcal{H}\left(J_{z}\right)\right],
$$

where $\rho\left(T_{\text {eff }}\right)$ is the equilibrium density matrix at temperature $T_{\text {eff }}$ :

$$
\rho\left(T_{\text {eff }}\right)=\frac{e^{-\mathcal{H}\left(J_{z}\right) / T_{\text {eff }}}}{\operatorname{Tr}\left[e^{\left.-\mathcal{H}\left(J_{z}\right) / T_{\text {eff }}\right]}\right.}
$$

This temperature is eventually averaged over disorder realizations in the cases $(I)-(I I)-(I I I)$.

In Fig. 5 we show the effective temperature as a function of the initial value of the anisotropy for a system of $L=12$ sites, quenched toward $J_{z}=0.5$. As it is apparent, $T_{\text {eff }}$ is monotonically increasing with $\left|J_{z}-J_{z 0}\right|$. In
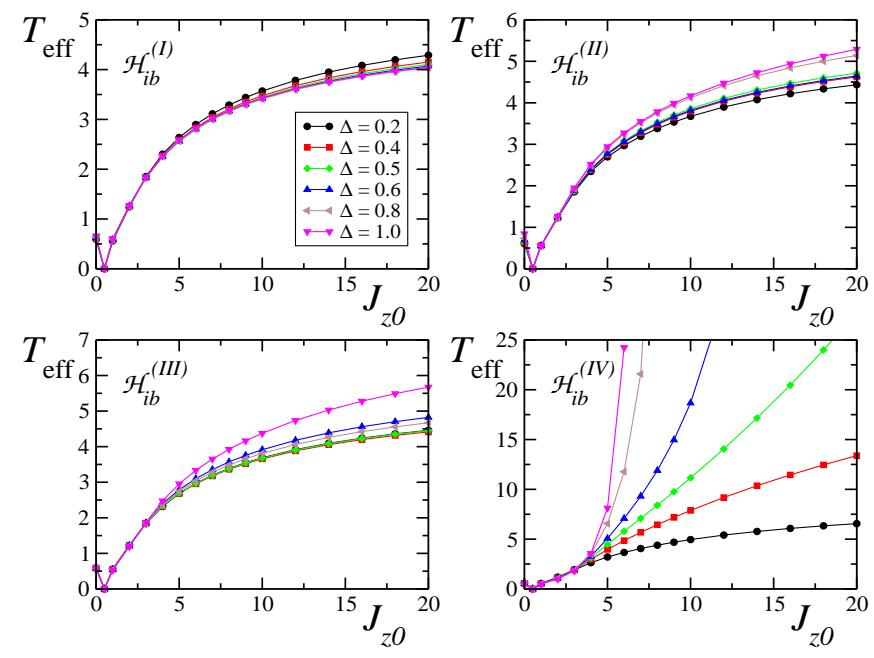

Figure 5: (color online). Effective temperature in the XXZ model with an integrability-breaking perturbation, after a quench in the anisotropy parameter toward a value of $J_{z}=$ 0.5. The values of $J_{z 0}$ in the $x$-axis denote the initial anisotropies, and stand for different initial conditions [that is, the ground states of the Hamiltonian $\left.\mathcal{H}\left(J_{z 0}\right)\right]$. Data are for $L=12$ sites; in all panels, except the lower right one, averages are performed over 200 disorder instances.

the first three cases, the effective temperature saturates for large values of $J_{z 0}$, because the initial ground state $\left|\psi_{0}\right\rangle$ tends toward the antiferromagnetic Néel state (for $J_{z} \gg 1$ and $\Delta \lesssim 1$ the effective temperature is around $T_{\text {eff }} \sim 5$, thus meaning that the states probed are located in the lower central part of the band) ${ }^{31}$.

\section{B. Thermalization of correlation functions}

We are now ready to test the relation between delocalization and thermalization after a quench by studying the long-time asymptotics of two-spin correlators, constructed as expectation values of

$$
n_{k}^{\alpha} \equiv \frac{1}{L} \sum_{j, l=1}^{L} e^{2 \pi i(j-l) k / L} \sigma_{j}^{\alpha} \sigma_{l}^{\alpha}, \quad(\alpha=x, z) .
$$

In particular, we compare the expectation value in the canonical ensemble at the corresponding $T_{\text {eff }}$ :

$$
n_{T_{\text {eff }}}^{\alpha}(k) \equiv\left\langle n_{k}^{\alpha}\right\rangle_{T_{\text {eff }}}=\operatorname{Tr}\left[\rho\left(T_{\text {eff }}\right) n_{k}^{\alpha}\right],
$$

with the asymptotic value that is reached after the quench, calculated from the diagonal ensemble ${ }^{10}$ :

$$
n_{Q}^{\alpha}(k) \equiv \lim _{t \rightarrow \infty}\left\langle\psi(t)\left|n_{k}^{\alpha}\right| \psi(t)\right\rangle=\sum_{i}\left|c_{i}\right|^{2}\left\langle\phi_{i}\left|n_{k}^{\alpha}\right| \phi_{i}\right\rangle,
$$

where $|\psi(t)\rangle=e^{-i \mathcal{H}\left(J_{z}\right) t}\left|\psi_{0}\right\rangle$ is the state of the system at time $t$, while $c_{i}=\left\langle\phi_{i} \mid \psi_{0}\right\rangle$ is the scalar product between the state $\left|\psi_{0}\right\rangle$ and the eigenstates $\left|\phi_{i}\right\rangle$ of the final Hamiltonian $\mathcal{H}\left(J_{z}\right)$. 
The observables we consider here correspond to two completely different scenarios in terms of the system quasiparticles, being $n_{k}^{x}$ a local operator while $n_{k}^{z}$ a nonlocal one. We recall that, in this context, local and nonlocal operators refer to the structure of their matrix elements on the basis of quasi-particles: local means that the operator couples a finite number of states, while nonlocal that it couples all states 13 . While correlators in the $x$-direction are always well reproduced by an effective thermal ensemble, correlators in the $z$-direction appear to be more sensitive to the breaking of integrability. This is seen quite clearly in Fig. 6, where we plot the correlators $n_{k}^{\alpha}$ averaged in the diagonal (black circles) and in the canonical (red squares) ensembles, both along the $x$-axis (upper panels) and along the $z$-axis (lower panels). The parameters are chosen in such a way as to have the system close to integrability, with a significant delocalization in Fock space still not present. Two different behaviors for $n^{x}(k)$ and $n^{z}(k)$ are apparent, with differences clearly emerging at the peaks $k=\pi$, where boundary effects are less pronounced. One can qualitatively see that, while discrepancies between the two ensembles are well visible in $n^{z}(k)$, they are suppressed in $n^{x}(k)$. Therefore, in a quasi-integrable regime only $n^{x}$ behaves thermally, while $n^{z}$ does not. As stated above, this reflects the intrinsic difference between nonlocal/local operators with respect to the quasiparticles, which emerges only for the cases in which the system itself is not able to properly hybridize the quasiparticle states within the microcanonical shell. Here we stress however that the classification of the operators is in general a subtle issue. In the model we considered in this work, it has been possible by analyzing the XX limit $\left(J_{z}=0\right)$ and the low-energy sector of the critical phase 32 .

A quantitative measure of the degree of thermalization is given by the absolute discrepancy between the diagonal and the canonical ensemble predictions:

$$
\delta n_{k}^{\alpha}=\left|n_{Q}^{\alpha}(k)-n_{T_{\text {eff }}}^{\alpha}(k)\right| .
$$

In order to elucidate the drastically different behavior between integrable and non-integrable systems, in Fig. 7 we plot $\delta n_{k}^{\alpha}$ at the peak $k=\pi$ where discrepancies are larger, as a function of the disorder amplitude $\Delta$. We observe that $\delta n_{\pi}^{x}$ is more than one order of magnitude smaller than $\delta n_{\pi}^{z}$, indicating a closely thermal behavior, while $\delta n_{\pi}^{z}$ shows a sharp decrease as integrability is progressively broken by increasing $\Delta$, towards a minimum value at $\bar{\Delta}$. The scaling with the dimension $L$ of the chain, shown in the upper left panel of Fig. (7), confirms our predictions. While the behavior of $\delta n_{\pi}^{x}$ is independent on the system size, the decrease of $\delta n_{\pi}^{z}$ as a function of $\Delta$ is more pronounced on increasing $L$. Due to the numerical limitations of exact diagonalization, we cannot rule out the possibility that, in the thermodynamic limit, the integrable to non-integrable transition for low perturbation strengths occurs at $\Delta^{*}=0$ in all the cases analyzed here $(I)-(I V)$. However, for the considered sizes, we found that the local observable $\delta n_{\pi}^{z}$ thermalizes the
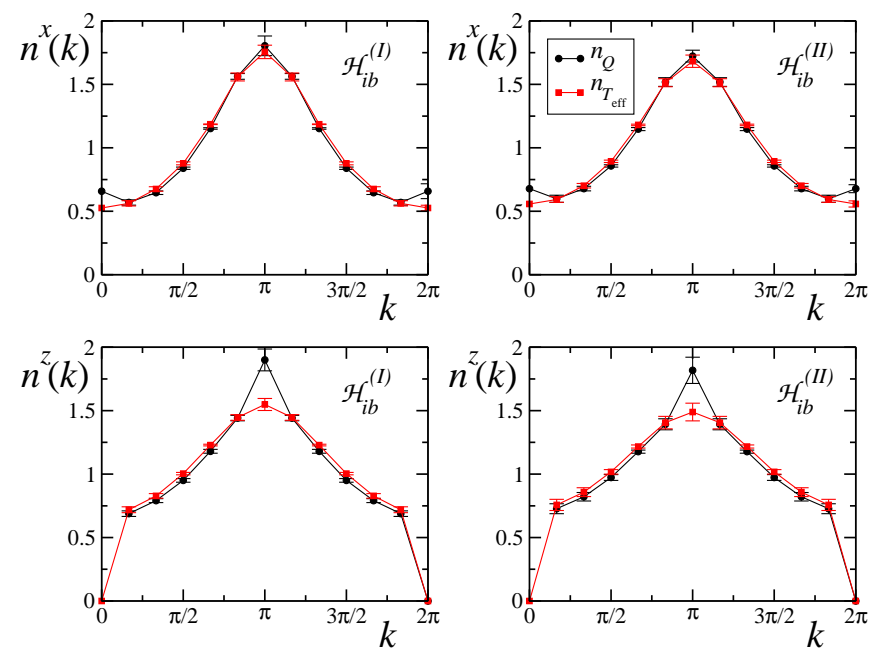

Figure 6: (color online). Comparison between the diagonal and canonical expectation value of the two-spin correlation function $n^{x}(k)$ (upper panels) and $n^{z}(k)$ (lower panels) as a function of the momentum $k$. Data are for a quench from $J_{z 0}=10$ to $J_{z}=0.5$ and two kinds of integrability-breaking perturbation $[(I)$ in left panels, and $(I I)$ in right panels], with disorder intensity $\Delta=0.4$.
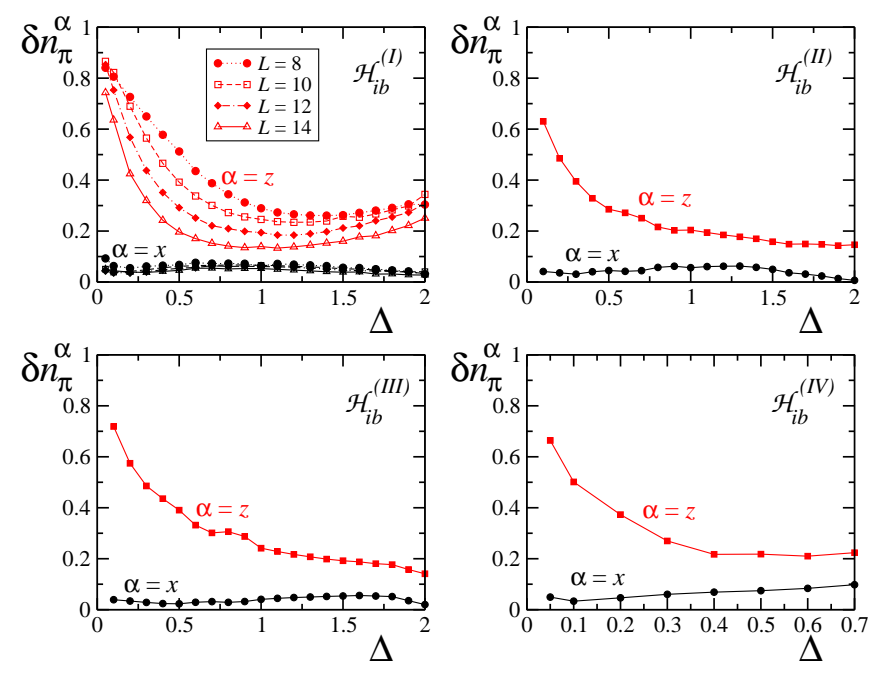

Figure 7: (color online). Discrepancies $\delta n_{\pi}^{x}$ (black circles) and $\delta n_{\pi}^{z}$ (red squares) between the diagonal and the canonical ensemble predictions, for a quench from $J_{z 0}=10$ to $J_{z}=0.5$. Data in the upper left panel $(I)$ are for different system sizes as depicted in the caption, while in the other panels are for $L=12$. In the cases of perturbations involving disorder, $(I)$ $(I I)-(I I I)$, averages over 200 instances are performed.

best at around $\bar{\Delta} \sim 1$ for model $(I)$. It is now crucial to observe that around this point, as noticed in the previous sections, the diffusive nature of the eigenstates is pronounced. The upper right and the lower left panels seem to locate the optimal thermalization point for models $(I I)$ and $(I I I)$ at a slightly larger value of $\Delta$, while 
the lower right panel shifts it to slightly smaller values for model $(I V)$. In all cases it is however true that at these points $\xi \simeq N_{[E, E+W]}$, making a direct connection between good thermalization and diffusive nature of the eigenstates. Notice also that, for $\Delta \gtrsim \bar{\Delta}, \delta n_{\pi}^{z}$ necessarily has to increase again, in agreement with the fact that $\Delta \simeq \bar{\Delta}$ is the point where the non-integrable behavior is most pronounced and that for large values of $\Delta$ the system tends toward another integrable limit $\underline{33}$. In analogy with previous studies, the different sensitivity to integrability of correlators in different spin directions can be qualitatively understood as a consequence of the fact that $\sigma^{z}$ is a local operator in quasi-particle space while $\sigma^{x}$ is a non-local one $\underline{\underline{13}}$.

\section{SUMMARY}

In conclusion, we discussed thermalization and integrability-breaking in the dynamics after a quench of a quantum XXZ Heisenberg spin chain in presence of an integrability-breaking term. We have shown that, if one wants to know when and how an interacting many-body system thermalizes, one should study the corresponding many-body localization/delocalization transition in quasi-particle space. Thermalization should occur when the relevant typical states spread diffusively on an exponential number of states lying in the microcanonical energy shell. We point out that our picture is valid as long as the integrability-breaking term acts homogeneously in the quasiparticle space, in such a way as to induce ergodicity over all the relevant Hilbert space. For generic dynamic systems there may be regions of the phase space which are non chaotic, so that their quantum versions produce entropy at a non-uniform rate given by the local Lyapunov exponents 34 . In this case more complex scenarios for the approach to equilibrium may arise.

\section{Acknowledgments}

We thank B. Altshuler, V. Kravtsov and D. Huse for fruitful discussions and useful comments on the manuscript. We also benefited from discussions with T. Caneva, M. Müller, G. Mussardo, A. Nersesyan, T. Prosen, A. Scardicchio, and M. Žnidarič.
1 J. von Neumann, Z. Phys. 57, 30 (1929).

2 W. Pauli and M. Fierz, Z. Phys. 106, 572 (1937).

3 P. Mazur, Physica 43, 533 (1968); E. Barouch and M. Dresden, Phys. Rev. Lett. 23, 114 (1969); M. Suzuki, Physica 51, 277 (1970).

4 A. Peres, Phys. Rev. A 30, 504 (1984).

5 J. M. Deutsch, Phys. Rev. A 43, 2046 (1991); M. Srednicki, Phys. Rev. E 50, 888 (1994).

6 S. Åberg, Phys. Rev. Lett. 64, 3119 (1990); Ph. Jacquod and D. L. Shepelyansky, Phys. Rev. Lett. 79, 1837 (1997); G. Benenti, G. Casati, and D. L. Shepelyansky, Eur. Phys. J D 17, 265 (2001).

7 T. Kinoshita, T. Wenger, and D. S. Weiss, Nature 440, 900 (2006).

8 M. Rigol, V. Dunjko, V. Yurovsky, and M. Olshanii, Phys. Rev. Lett. 98, 050405 (2007).

9 M. A. Cazalilla, Phys. Rev. Lett. 97, 156403 (2006); P. Calabrese and J. Cardy, Phys. Rev. Lett. 96, 136801 (2006); J. Stat. Mech. (2007) P06008; M. Cramer, C. M. Dawson, J. Eisert, and T. J. Osborne, Phys. Rev. Lett. 100, 030602 (2008); T. Barthel and U. Schollwöck, Phys. Rev. Lett. 100, 100601 (2008); M. Eckstein and M. Kollar, Phys. Rev. Lett. 100, 120404 (2008); D. M. Gangardt and M. Pustilnik, Phys. Rev. A 77, 041604(R) (2008); D. Fioretto and G. Mussardo, New J. Phys. 12, 055015 (2010).

10 M. Rigol, V. Dunjko, and M. Olshanii, Nature 452, 854 (2008); M. Rigol, Phys. Rev. Lett. 103, 100403 (2009); M. Rigol and L. F. Santos, Phys. Rev. A 82, 011604(R) (2010).

11 C. Kollath, A. M. Läuchli, and E. Altman, Phys. Rev. Lett. 98, 180601 (2007). S. R. Manmana, S. Wessel, R. M. Noack, and A. Muramatsu, Phys. Rev. Lett. 98, 210405 (2007); M. Eckstein, M. Kollar, and P. Werner, Phys. Rev. Lett. 103, 056403 (2009).
12 G. Roux, Phys. Rev. A 79, 021608(R) (2009); G. Biroli, C. Kollath, and A. M. Läuchli, Phys. Rev. Lett. 105, 250401 (2010).

13 D. Rossini, A. Silva, G. Mussardo, and G. E. Santoro, Phys. Rev. Lett. 102, 127204 (2009); D. Rossini, S. Suzuki, A. Silva, G. Mussardo, and G. E. Santoro, Phys. Rev. B 82, 144302 (2010).

14 D. M. Basko, I. L. Aleiner, and B. L. Altshuler, Ann. Phys. (NY) 321, 1126 (2006).

15 I. V. Gornyi, A. D. Mirlin, and D. G. Polyakov, Phys. Rev. Lett. 95 , 206603 (2005).

16 B. L. Altshuler, Y. Gefen, A. Kamenev, and L. S. Levitov, Phys. Rev. Lett. 78, 2803 (1997).

17 D. E. Logan and P. G. Wolynes, J. Chem. Phys. 93, 4994 (1990).

18 A. Pal and D. A. Huse, Phys. Rev. B 82, 174411 (2010).

19 M. Žnidarič, T. Prosen, and P. Prelov šek, Phys. Rev. B 77, 064426 (2008).

${ }^{20}$ V. Oganesyan and D. Huse, Phys. Rev. B 75, 155111 (2007), V. Oganesyan , A. Pal, D .A. Huse, Phys. Rev. B 80, 115104 (2009).

21 Localization is intended in the space of quasi-particles, and not necessarily in real space.

22 M. V. Berry, J. Phys. A: Math. Gen. 10, 2083 (1977).

23 Notice that in general it is possible to have non-ergodic, but extended states (see Ref. 16 and Refs. therein).

24 F. D. M. Haldane, Phys. Rev. Lett. 47, 1840 (1981).

25 C. A. Doty, D. S. Fisher, Phys. Rev. B 452167 (1992)

${ }^{26}$ While for the parameters used in this paper the low-lying eigenstates are localized in the thermodynamic limit, in the following we consider systems sizes smaller than the localization length.

27 F. Haake, Quantum Signatures of chaos (Springer-Verlag, Berlin, 1991). 
28 M. Di Stasio and X. Zotos, Phys. Rev. Lett. 74, 2050 (1995).

29 Y. Avishai, J. Richert, and R. Berkovits, Phys. Rev. B 66, 052416 (2002); K. Kudo and T. Deguchi, Phys. Rev. B 69, 132404 (2004).

${ }^{30}$ W. G. Brown, L. F. Santos, D. J. Starling, and L. Viola, Phys. Rev. E 77, 021106 (2008); F. Dukesz, M. Zilbergerts, and L. F. Santos, New J. Phys. 11 (2009) 043026.

31 The situation is slightly different when a static next-tonearest neighbor coupling is added (lower right panel). In this case, for large values of $\Delta$ and sufficiently large $J_{z 0}$, equation (9) does not admit a physically meaningful solution.

32 N. Nagaosa, Quantum Field Theory in Strongly Correlated Electronic Systems (Springer, Berlin, 1999).

33 The raising of $\delta n_{\pi}^{z}$ for $\Delta \gtrsim \bar{\Delta}$ is apparent for the case $(I)$, as shown in the upper left panel of Fig. 7 For the other cases we do not show pertinent data, as this is not the main point we concern with.

34 D. Monteoliva and J. P. Paz, Phys. Rev. Lett. 85, 3373 (2000). 